\title{
Assessing disability and relapses in multiple sclerosis on tele-neurology
}

\author{
Marcello Moccia ${ }^{1}$ (D) Roberta Lanzillo $^{1}$ - Vincenzo Brescia Morra ${ }^{1} \cdot$ Simona Bonavita ${ }^{2} \cdot$ Gioacchino Tedeschi $^{3}$. \\ Letizia Leocani ${ }^{4,5,6}$ - Luigi Lavorgna ${ }^{3}$ - on behalf of the Digital Technologies Web and Social Media Study Group of the \\ Italian Society of Neurology
}

Received: 28 April 2020 / Accepted: 9 May 2020 / Published online: 21 May 2020

(C) Fondazione Società Italiana di Neurologia 2020

\begin{abstract}
Background As a consequence of the coronavirus disease 2019 (COVID-19) pandemic, a large amount of consultations will be delivered through tele-medicine, especially for diseases causing chronic disability and requiring immunomodulatory treatments, such as multiple sclerosis (MS).

Methods We have hereby reviewed available tools for tele-neurology examination in MS, including components of neurological examination that can be assessed through video, patient-reported outcome measures (PROMs), and digital technology. Results Overall, we have suggested a battery for assessing MS disability and relapses on tele-medicine, which brings together conventional examination, PROMs (e.g., Patient Determined Disease Steps, MS Impact Scale), and cognitive tests (Symbol Digit Modalities Test) that can be delivered remotely and in multiple languages.

Discussion The use of common tools for neurological examination could improve tele-neurology practice for both general neurologists and MS specialists, and quality of care for people with MS.
\end{abstract}

Key words Multiple sclerosis · Tele-neurology $\cdot$ Tele-medicine $\cdot$ COVID

From the end of December 2019, coronavirus disease 2019 (COVID-19) has progressively spread through the world, and,

Marcello Moccia

marcello.moccia@unina.it; moccia.marcello@gmail.com

1 Multiple Sclerosis Clinical Care and Research Centre, Department of Neuroscience, Reproductive Science and Odontostomatology, Federico II University of Naples, Via Sergio Pansini 5, 80131 Naples, Italy

2 Department of Medical, Surgical, Neurological, Metabolic and Aging Sciences, II Clinic of Neurology, University of Campania "Luigi Vanvitelli", Naples, Italy

3 Department of Medical, Surgical, Neurological, Metabolic and Aging Sciences, University of Campania "Luigi Vanvitelli", Naples, Italy

4 Department of Neurorehabilitation, IRCCS San Raffaele Hospital, Milan, Italy

5 Experimental Neurophysiology Unit, Division of Neuroscience, Institute of Experimental Neurology (INSPE), San Raffaele Scientific Institute, Milan, Italy

6 University Vita-Salute San Raffaele, Milan, Italy on March 11, the World Health Organization declared COVID-19 as a pandemic [1]. Healthcare resources and staff have been immediately redeployed towards COVID-19 management, with consultations for chronic diseases being canceled, postponed, or converted to tele-medicine [1,2]. However, in the upcoming months, COVID-19 is expected to circulate and, thus, a large amount of consultations will be converted to tele-medicine, especially for diseases causing chronic disability and requiring immunomodulatory treatments, such as multiple sclerosis (MS) [3].

MS is characterized by acute-onset neurological symptoms (relapses), and chronic disability, which are primarily evaluated on neurological examination [4]. Following the COVID-19 emergency, the American Academy of Neurology (AAN) has published recommendations for implementing a tele-medicine service, suggesting that general neurological examination is feasible remotely, but with some caveats [5]. For instance, there are difficulties in comprehensive examination of eyes (i.e., fundoscopy), neuro-muscolar components (i.e., reflexes), and vestibular system (i.e., Dix-Hallpike maneuver), while the evaluation of other neurological systems depends on the teleneurology setup (i.e., space enough for gait examination), and 
Table 1 Suggested battery for assessing MS disability and relapses on tele-medicine. The table shows suggested tools for assessing MS disability and relapses on tele-medicine. In particular, PDDS and MSIS-
29 could be administered before tele-consultation, and, then, integrated with history, taking more detailed neurological examination, and cognitive screening (SDMT), as necessary

\begin{tabular}{|c|c|c|c|}
\hline & Tool & Advantages & Limitations \\
\hline \multirow[t]{2}{*}{$\begin{array}{l}\text { Before } \\
\quad \text { consultation }\end{array}$} & PDDS & $\begin{array}{l}\text { Available in multiple languages } \\
\text { Available for self-assessment } \\
\text { Already validated online }\end{array}$ & $\begin{array}{l}\text { Limited representation of brainstem and } \\
\text { cognitive function }\end{array}$ \\
\hline & MSIS-29 & $\begin{array}{l}\text { Available in multiple languages } \\
\text { Available for self-assessment } \\
\text { Already validated online }\end{array}$ & $\begin{array}{l}\text { To be integrated with more detailed } \\
\text { scales/ } \\
\text { questionnaires, as necessary }\end{array}$ \\
\hline \multirow[t]{4}{*}{$\begin{array}{l}\text { During } \\
\quad \text { consultation }\end{array}$} & Full neurological examination & Flexible (depending on patients' symptoms) & $\begin{array}{l}\text { Specific setup } \\
\text { Caregiver to help } \\
\text { Time consuming }\end{array}$ \\
\hline & $\begin{array}{l}\text { Examination of brainstem } \\
\text { function }\end{array}$ & Largely feasible remotely & To be integrated with PDDS \\
\hline & EDSS & $\begin{array}{l}\text { Current standard in MS clinical practice and } \\
\text { research }\end{array}$ & $\begin{array}{l}\text { Specific setup } \\
\text { Caregiver to help } \\
\text { Time consuming }\end{array}$ \\
\hline & SDMT & $\begin{array}{l}\text { Suitable for all languages } \\
\text { Available for self-assessment } \\
\text { Already validated online }\end{array}$ & $\begin{array}{l}\text { More detailed tests for diagnosis of } \\
\text { cognitive impairment }\end{array}$ \\
\hline
\end{tabular}

the availability of a caregiver to help (i.e., sensory examination) [5]. Remote examination could be further implemented with the use of digital technology, such as smartphone accelerometers for gait, posture, and balance assessment [6]. Similarly, over the past years, different studies have already explored the feasibility of remote counseling in MS, but have focused on specific aspects of the disease (e.g., treatment adherence, mental health) [7-9]. As such, there is no general agreement on MS-specific neurological examination on teleconsultations. Hereby, we have briefly reviewed available tools for remote evaluation of disability progression and relapses in MS, and have suggested a battery for tele-neurology examination in MS.

In MS, different neurological systems are combined into the Expanded Disability Status Scale (EDSS), which is the standard tool for clinical practice, observational studies, and clinical trials [4]. The possibility of assessing the EDSS remotely has been explored, but would require to use an "inhome neuro kit" with vision card, tuning fork, pin, cotton swab, and alcohol swab [10], to have a caregiver helping with the examination $[9,10]$, and to combine records from wearable devices (e.g., accelerometer-based step counts) [7, 9]. As such, remote EDSS faces some organizational issues, which would be difficult to overcome in the short term, but could be considered when strictly required (e.g., patients included in research protocols). Also, data from wearable devices should be interpreted cautiously, considering that the occurrence of new or recurrent neurological symptoms may not necessarily correspond to reduced physical activity in patients already self-isolating at home.

On the contrary, patient-reported outcome measures (PROMs) would remain entirely feasible online. The Patient
Determined Disease Steps (PDDS) has been specifically developed as a PROM of MS disability and has been validated in multiple languages, and also for online administration [11]. The PDDS is strongly correlated to EDSS, and especially to visual, pyramidal, cerebellar, sensory, bowel/bladder, and ambulatory functional systems, leaving brainstem and cerebral functions relatively unexplored [12]. As such, if patients are required to fill in the PDDS before tele-consultations, remote examination could specifically focus on brainstem function (e.g., ocular movements, trigeminal damage, hearing loss, dysarthria, and dysphagia), which is possibly easier to examine remotely or, at least, on history taking. Looking at cerebral function, different neuropsychological tests have been assessed for remote use [13]. Among them, the Symbol Digit Modalities Test (SDMT), which measures attention and processing speed, holds strong correlates to disability progression, and has been suggested as a screening tool for cognitive impairment in MS [14]. The oral version of the SDMT has already been validated for remote use (e.g., online or during functional-MRI acquisitions), is ideally suitable for all languages [14], and, thus, could be easily used on teleneurology.

Relapses are defined as the occurrence of new or recurrent neurological abnormalities, which are separated by $\geq 30$ days from the onset of the preceding event, last for $\geq 24 \mathrm{~h}$, and occur without fever or infection $[4,15]$. Thus, if some elements are relatively easy to evaluate on remote history taking (e.g., timeline, concomitant fever or symptoms of infection), neurological abnormalities would need to be detected as suggested above, along with other neurological tests in relation to reported symptoms. However, relapses also affect quality of life and activities of daily living [15], and, thus, the inclusion 
of MS-specific PROMs measuring physical and psychological health could support the assessment of relapse severity. For instance, the MS Impact Scale (MSIS-29) has been used in clinical trials to evaluate recovery from relapses [15], and has been tested for remote administration [16]. Alternative scales could be considered depending on prevalent patients' symptoms (e.g., mood, fatigue). However, in-person examination and/or MRI should be considered for cases where a relapse cannot be fully ruled out, in order to make timely treatment decisions [4].

Overall, we have suggested a battery for MS assessment in tele-neurology, which strongly leverages patients' empowerment and PROMs (Table 1). In particular, we recommend MS patients fill in the PDDS and the MSIS-29 before the teleconsultation, so that neurologists have a full view on the overall physical and psychological status. Then, during the teleconsultation, neurologists can focus on history taking and examination of brainstem function and other neurological systems, as required by patients' symptoms. Finally, if necessary, the examiner could administer the oral version of the SDMT by showing the test form on patients' screen. Alternatively, patients could be provided with PDDS, MSIS-29, and an electronic version of the SDMT [17], for self-assessment, and, then, request tele-neurology if necessary. The PDDS, the MSIS-29, and the SDMT could be integrated to videoconsultation platforms or electronic health record systems, or be implemented using other online tools, such as Google Forms.

Limitations of tele-neurology include the availability of technology to deliver tele-consultations (e.g., webcam quality, high-speed connection), which need to be accounted for by policymakers to avoid healthcare inequalities.

In conclusion, though the medical management of COVID19 is the current healthcare priority, chronic diseases should not be left unattended, and the use of common tools for neurological examination could improve tele-neurology practice, and quality of care for people with MS.

Acknowledgements We acknowledge the contribution of the Digital Technologies, Web and Social Media Study Group of Italian Neurological Society (SIN): Luigi Lavorgna (Chair), Letizia Leocani (Co-Chair), Gioacchino Tedeschi, Giovanni Mancardi, Alessandro Padovani, Simona Bonavita, Francesco Brigo, Marinella Clerico, Eleonora Cocco, Roberta Lanzillo, Marcello Moccia, Luca Prosperini, Maria Laura Stromillo, Anna Maria Repice, Gianmarco Abbadessa, Alberto Lerario, Francesca Trojsi, Rosa Iodice, Sebastiano Bucello, Pietro Annovazzi, Antonio De Martino, Alessandro Bombaci, Francesco Iodice, and Francesco Di Lorenzo.

\section{Compliance with ethical standards}

\section{Conflict of interest None}

\section{References}

1. Bonavita S, Tedeschi G, Atreja A, Lavorgna L (2020) Digital triage for people with multiple sclerosis in the age of COVID-19 pandemic. Neurol Sci

2. Waldman G, Mayeux R, Claassen J et al (2020) Preparing a neurology department for SARS-CoV-2 (COVID-19): early experiences at Columbia University Irving Medical Center and the New York Presbyterian Hospital in New York City. Neurology.

3. Brownlee W, Bourdette D, Broadley S et al (2020) Treating multiple sclerosis and neuromyelitis optica spectrum disorder during the COVID-19 pandemic. Neurology.

4. Tur C, Moccia M, Barkhof F et al (2018) Assessing treatment outcomes in multiple sclerosis trials and in the clinical setting. Nat Rev Neurol 14:75-93

5. American Academy of Neurology (2020) Telemedicine and remote care. In: AAN COVID-19 Neurol. Resour. Cent. https://www.aan. com/tools-and-resources/practicing-neurologists-administrators/ telemedicine-and-remote-care/. Accessed 19 Apr 2020

6. Galán-Mercant A, Cuesta-Vargas AI (2014) Mobile Romberg test assessment (mRomberg). BMC Res Notes 7:640

7. Lavorgna L, Brigo F, Moccia M et al (2018) e-Health and Multiple Sclerosis: an update. Mult Scler 24:1657-1664

8. Robb JF, Hyland MH, Goodman AD (2019) Comparison of telemedicine versus in-person visits for persons with multiple sclerosis: a randomized crossover study of feasibility, cost, and satisfaction. Mult Scler Relat Disord 36:101258

9. Yeroushalmi S, Maloni H, Costello K, Wallin M (2019) Telemedicine and multiple sclerosis: a comprehensive literature review Samuel. J Telemed Telecare

10. Bove R, Bevan C, Crabtree E et al (2019) Toward a low-cost, inhome, telemedicine-enabled assessment of disability in multiple sclerosis. Mult Scler 25:1526-1534

11. Lavorgna L, Sparaco M, Esposito S et al (2017) Validity and reproducibility of the Italian version of the patient determined disease steps scale in people with multiple sclerosis. Mult Scler Relat Disord 18:173-176

12. Learmonth YC, Motl RW, Sandroff BM et al (2013) Validation of patient determined disease steps (PDDS) scale scores in persons with multiple sclerosis. BMC Neurol 13:37

13. Wojcik C, Beier M, Costello K et al (2019) Computerized neuropsychological assessment devices in multiple sclerosis: a systematic review. Mult Scler 25:1848-1869

14. Silva PHR, Spedo CT, Barreira AA, Leoni RF (2018) Symbol Digit Modalities Test adaptation for Magnetic Resonance Imaging environment: a systematic review and meta-analysis. Mult Scler Relat Disord 20:136-143

15. Chataway J, Porter B, Riazi A et al (2006) Home versus outpatient administration of intravenous steroids for multiple-sclerosis relapses: a randomised controlled trial. Lancet Neurol 5:565-571

16. Hobart J, Lamping D, Fitzpatrick R et al (2001) The Multiple Sclerosis Impact Scale (MSIS-29): a new patient-based outcome measure. Brain 124:962-973

17. Bigi S, Marrie RA, Till C et al (2017) The computer-based Symbol Digit Modalities Test: establishing age-expected performance in healthy controls and evaluation of pediatric MS patients. Neurol Sci 38:635-642

Publisher's note Springer Nature remains neutral with regard to jurisdictional claims in published maps and institutional affiliations.

Ethical approval None 